\title{
Acoustical Studies of some Chalcones in DMF and DMSO Solutions
}

\author{
Shipra Baluja*, Pravin Kasundra \\ Physical Chemistry Laboratory, Department of Chemistry, Saurashtra University, \\ Rajkot - 360005 India \\ *E-mail address: shipra_baluja@rediffmail.com
}

\begin{abstract}
This work reports densities, speeds of sound, and isentropic compressibility of the solutions of some synthesized chalcones in N,N-dimethylformamide and dimethyl sulphoxide solutions of various concentrations at $308.15 \mathrm{~K}$ with a view to understand molecular interactions in these solutions.
\end{abstract}

Keywords: Chalcones; sound velocity; isentropic compressibility

\section{INTRODUCTION}

Literature survey shows that because of its non destructive nature, ultrasonic waves have been used in various industries for various processes like dyeing ${ }^{1}$, cleaning ${ }^{2}$, design $^{3}$, bleaching $^{4}$, food processing ${ }^{5}$, organic synthesis ${ }^{6,7}$ etc. Further, it is also used in medical fields ${ }^{8-10}$.

The ultrasonic measurements have also been used to study molecular interactions in various pure liquids ${ }^{11}$, liquid mixtures ${ }^{12,13}$ and solutions of organic and inorganic compounds $^{14-16}$, polymers ${ }^{17}$, amino acids ${ }^{18}$, drugs ${ }^{19}$ etc. The thermodynamic parameters calculated through ultrasonic velocity measurements can give idea about intermolecular attraction between molecules (solute-solute or solute-solvent) ${ }^{28}$.

In our laboratory, the ultrasonic and physicochemical studies of some organic compounds like Schiff bases ${ }^{20}$, triazoles $^{21}$, dihydropyrimidines ${ }^{22}$ etc. have been studied in different solvents. In continuation of our previous work, in the present paper, ultrasonic studies of some chalcones base have been studied in $\mathrm{N}, \mathrm{N}$ dimethylformamide and dimethylsulphoxide solutions of various concentrations at $303.15 \mathrm{~K}$ with a view to understand molecular interactions in these solutions.

\section{EXPERIMENTAL}

The solvents N, N-dimethylformamide and dimethylsulphoxide were of analytical grade and were distilled by the reported procedure ${ }^{23}$. The purities of these solvents values were checked by a gas-liquid chromatography and were found to be $99.5 \%$ and $99.6 \%$ for $\mathrm{N}, \mathrm{N}$ dimethylformamide and dimethyl sulphoxide respectively. The solvents were degassed and dried over molecular sieves (Union Carbide, type $0.4 \mathrm{~nm}$ ). 
The chalcones (ANC-1 to ANC-9) were synthesized in our laboratory and were recrystalized before use. The physical constants of all the synthesized chalcones are given in Table 1. The general structure of chalcone is given in Figure 1.

The densities, viscosities and ultrasonic velocities of pure solvents and solutions of chalcones of different concentrations were measured at $303.15 \mathrm{~K}$ by using pyknometer, an Ubbelohde suspended level viscometer and single frequency ultrasonic interferometer (Mittal Enterprises, Model No F81) operating at $2 \mathrm{MHz}$, with the uncertainties of $0.0001 \mathrm{~g} / \mathrm{cm}^{3}$, \pm $0.06 \%$ and $0.01 \%$ respectively. The temperature was maintained by an electronically controlled thermostatic water bath (NOVA NV-8550 E). The uncertainty of temperature was $\pm 0.1^{\circ} \mathrm{C}$.

All the solutions were prepared by using a Mettler Toledo AB204-S balance with a precision of $\left(1 \times 10^{-4}\right) \mathrm{g}$.

\section{RESULTS AND DISCUSSION}

Table 2 shows the experimental data of density $(\rho)$, viscosity $(\eta)$ and sound velocity $(U)$ of pure solvents and solutions of chalcones in DMF and DMSO solutions at 303.15 K. From these experimental data, various acoustical parameters like specific acoustical impedance $(Z)$, isentropic compressibility $\left(\kappa_{S}\right)$, inter molecular free length $\left(L_{f}\right)$, Rao's molar sound function $\left(R_{m}\right)$, Vander Waals constant $(b)$, relaxation strength $(r)$, internal pressure $(\pi)$, apparent molar compressibility $\left(\phi_{k}\right)$ etc., were evaluated using the equations reported earlier ${ }^{20}$.

Some of the calculated acoustical parameters are given in Tables 3 and 4 for all the compounds in DMF and DMSO solutions respectively. Figure 2 shows the variation of ultrasound velocity $(U)$ with concentration in DMF and DMSO respectively. It is observed that in both the solvents, ultrasonic velocity $(U)$ increases non linearly with concentration for all the compounds. Whereas Figure 3 shows that intermolecular free length $\left(L_{f}\right)$ decreases with concentration. Thus, ultrasonic velocity is reverse of intermolecular free length $\left(L_{f}\right)$. The decrease of intermolecular free length means the distance between solute (compound) and solvent molecules decreases i.e., strong interaction between solvents and compound molecules exists in these solvents which causes ultrasonic velocity to increase.

This is further supported by isentropic compressibility $\left(\kappa_{S}\right)$ and relaxation strength $(r)$. The isentropic compressibility $\left(\kappa_{S}\right)$ of the solutions in both the solvents is also found to decrease with increase of concentration, as shown in Figure 4. Due to solute-solvent interactions in the system, compressibility of the solution decreases with the increase in concentration. This is further confirmed by decrease of relaxation strength $(r)$ and increase in specific impedance (Z) values (as reported in Tables 3 and 4). The Rao's molar sound function $\left(R_{m}\right)$ and Vander Waal's constant (b) are also observed to increase linearly (correlation coefficient 0.9985-0.9998) with concentration for all the compounds in both the solvents. The linear increase of these parameters shows absence of complex formation in these systems.

The internal pressure is the result of forces of attraction and repulsion between molecules in a solution. As Tables 3 and 4 shows that internal pressure $(\pi)$ decreases in both the solvents. This indicates that solute-solute interactions also exist in these solutions.

The predominance of solute-solvent interactions is further confirmed by evaluating some other parameters. The isentropic compressibility of all the solutions was also fitted to the following Bachem's relation ${ }^{24}$ : 


$$
\kappa_{s}=\kappa_{s}^{0}+A C+B C^{3 / 2}
$$

From the plot of $\left(\kappa_{s}-\kappa_{s}^{0}\right) / C$ verses $\sqrt{ } C$, values of $A$ and $B$ constants were evaluated from the intercept and slope respectively. $\kappa_{s}^{0}$ is the isentropic compressibility of pure solvent. Further, the apparent molar compressibility $\left(\phi_{\mathrm{k}}\right)$ of the solutions is fitted to Gucker's relation25:

$$
\phi_{k}=\phi_{k}^{o}+S_{k} \sqrt{ } C
$$

From the plot of $\phi_{k}$ verses $\sqrt{ } C, \phi_{k}^{o}$ and $S_{k}$ values are evaluated from the intercept and slope. $S_{k}$ is known as interaction parameter.

It is evident from Table 5 that in both DMF and DMSO solutions, $A$ and $\phi_{k}^{o}$, values are negative whereas $B$ and $S_{k}$ values are positive. The negative $A$ and $\phi_{k}^{o}$ confirms interaction between solvent and compound molecules. Further, the positive values of B and $S_{k}$ confirms the predominance of solute-solvent interactions in studied systems.

Thus, it is concluded that for the synthesized chalcones, although both solute-solute and solute-solvent interactions exist in these solutions, solute-solvent interactions dominate in both DMF and DMSO solutions.

\begin{tabular}{|c|c|c|c|c|c|}
\hline Code & $R$ & M.F. & M.W. & Yield (\%) & $R_{f}$ value* \\
\hline ANC-1 & $4-\mathrm{CH}_{3}, \mathrm{C}_{6} \mathrm{H}_{4}-$ & $\mathrm{C}_{24} \mathrm{H}_{18} \mathrm{O}$ & 322.40 & 62 & 0.46 \\
\hline ANC -2 & $4-\mathrm{OH}-\mathrm{C}_{6} \mathrm{H}_{4}-$ & $\mathrm{C}_{23} \mathrm{H}_{16} \mathrm{O}_{2}$ & 324.37 & 45 & 0.56 \\
\hline ANC -3 & 4-Br- $\mathrm{C}_{6} \mathrm{H}_{4-}$ & $\mathrm{C}_{23} \mathrm{H}_{15} \mathrm{BrO}$ & 387.27 & 69 & 0.49 \\
\hline ANC -4 & $4-\mathrm{F}-\mathrm{C}_{6} \mathrm{H}_{4-}^{-}$ & $\mathrm{C}_{23} \mathrm{H}_{15} \mathrm{FO}$ & 326.36 & 74 & 0.52 \\
\hline ANC -5 & $4-\mathrm{Cl}-\mathrm{C}_{6} \mathrm{H}_{4-}$ & $\mathrm{C}_{23} \mathrm{H}_{15} \mathrm{ClO}$ & 342.82 & 71 & 0.56 \\
\hline ANC-6 & $4-\mathrm{NO}_{2}-\mathrm{C}_{6} \mathrm{H}_{4}-$ & $\mathrm{C}_{23} \mathrm{H}_{15} \mathrm{NO}_{3}$ & 353.37 & 79 & 0.51 \\
\hline ANC -7 & $4-\mathrm{NH}_{2}-\mathrm{C}_{6} \mathrm{H}_{4-}$ & $\mathrm{C}_{23} \mathrm{H}_{17} \mathrm{NO}$ & 323.39 & 65 & 0.56 \\
\hline ANC -8 & $4-\mathrm{OCH}_{3}-\mathrm{C}_{6} \mathrm{H}_{4-}$ & $\mathrm{C}_{24} \mathrm{H}_{18} \mathrm{O}_{2}$ & 338.40 & 61 & 0.47 \\
\hline ANC -9 & $\mathrm{C}_{9} \mathrm{H}_{5} \mathrm{O}_{2-}$ & $\mathrm{C}_{26} \mathrm{H}_{16} \mathrm{O}_{3}$ & 376.40 & 59 & 0.58 \\
\hline
\end{tabular}

Table 1. Physical constants of synthesized Chalcones.

*Hexane: Ethyl acetate-6.5:3.5

Table 2. The density $(\rho)$, ultrasonic velocity $(U)$ and viscosity $(\eta)$ of Chalcones in DMF and DMSO.

\begin{tabular}{|c|c|c|c|c|c|c|}
\hline $\begin{array}{c}\text { Conc. } \\
M\end{array}$ & $\begin{array}{c}\text { Density } \\
\rho \\
\text { g.cm }\end{array}$ & $\begin{array}{c}\text { Velocity } \\
\text { U. } 10^{-5} \\
\text { cm.s-1 }\end{array}$ & $\begin{array}{c}\text { Viscosity } \\
\eta \cdot 10^{3} \\
\text { poise }\end{array}$ & $\begin{array}{c}\text { Density } \\
\rho \\
\text { g.cm }\end{array}$ & $\begin{array}{c}\text { Velocity } \\
\text { U. } 10^{-5} \\
\text { cm.s-1 }\end{array}$ & $\begin{array}{c}\text { Viscosity } \\
\eta \cdot 10^{3} \\
\text { poise }\end{array}$ \\
\hline \multicolumn{7}{|c|}{ DMF } \\
\hline \multicolumn{7}{|c|}{ ANC-1 } \\
\hline 0.00 & 0.9337 & 1.4308 & 7.2751 & 1.0846 & 1.4604 & 16.5573 \\
\hline 0.01 & 0.9372 & 1.4316 & 7.3778 & 1.0847 & 1.4608 & 16.9630 \\
\hline 0.02 & 0.9383 & 1.4328 & 7.4699 & 1.0848 & 1.4612 & 17.0657 \\
\hline 0.04 & 0.9399 & 1.4328 & 7.5423 & 1.085 & 1.4628 & 17.2434 \\
\hline 0.06 & 0.9408 & 1.4360 & 7.6611 & 1.0854 & 1.4628 & 17.3003 \\
\hline 0.08 & 0.9423 & 1.4368 & 7.7571 & 1.0858 & 1.4632 & 17.3527 \\
\hline
\end{tabular}




\begin{tabular}{|c|c|c|c|c|c|c|}
\hline 0.10 & 0.9438 & 1.4408 & 7.8894 & 1.0868 & 1.4664 & 17.5251 \\
\hline \multicolumn{7}{|c|}{ ANC-2 } \\
\hline 0.01 & 0.9363 & 1.4320 & 7.3152 & 1.0848 & 1.4680 & 17.8006 \\
\hline 0.02 & 0.9370 & 1.4348 & 7.3603 & 1.0849 & 1.4688 & 17.9631 \\
\hline 0.04 & 0.9372 & 1.4360 & 7.4373 & 1.0856 & 1.4732 & 18.1724 \\
\hline 0.06 & 0.9374 & 1.4388 & 7.4826 & 1.0865 & 1.4748 & 18.2886 \\
\hline 0.08 & 0.9384 & 1.4440 & 7.5700 & 1.0875 & 1.4752 & 18.3884 \\
\hline 0.10 & 0.9405 & 1.4488 & 7.6547 & 1.0887 & 1.4768 & 18.7221 \\
\hline \multicolumn{7}{|c|}{ ANC-3 } \\
\hline 0.01 & 0.9376 & 1.4320 & 7.3214 & 1.0849 & 1.4676 & 17.8758 \\
\hline 0.02 & 0.9391 & 1.4320 & 7.4206 & 1.0853 & 1.4720 & 18.1719 \\
\hline 0.04 & 0.9401 & 1.4336 & 7.4564 & 1.0866 & 1.4756 & 18.4376 \\
\hline 0.06 & 0.9407 & 1.4360 & 7.5647 & 1.0881 & 1.4828 & 19.0482 \\
\hline 0.08 & 0.9427 & 1.4380 & 7.6885 & 1.0922 & 1.4892 & 19.8600 \\
\hline 0.10 & 0.9491 & 1.4388 & 7.8292 & 1.0952 & 1.4936 & 20.1557 \\
\hline \multicolumn{7}{|c|}{ ANC-4 } \\
\hline 0.01 & 0.9376 & 1.4316 & 7.3373 & 1.0849 & 1.4648 & 17.0534 \\
\hline 0.02 & 0.9394 & 1.4324 & 7.4230 & 1.0863 & 1.4660 & 17.2641 \\
\hline 0.04 & 0.942 & 1.4344 & 7.4914 & 1.0876 & 1.4664 & 17.3492 \\
\hline 0.06 & 0.9451 & 1.4372 & 7.5961 & 1.0888 & 1.4696 & 17.5804 \\
\hline 0.08 & 0.9464 & 1.4428 & 7.7027 & 1.0906 & 1.4700 & 18.1683 \\
\hline 0.10 & 0.9493 & 1.4444 & 7.7786 & 1.0921 & 1.4700 & 18.4245 \\
\hline \multicolumn{7}{|c|}{ ANC-5 } \\
\hline 0.01 & 0.9374 & 1.4324 & 7.7088 & 1.0847 & 1.4636 & 16.8115 \\
\hline 0.02 & 0.9398 & 1.4336 & 7.7723 & 1.0848 & 1.4640 & 16.9738 \\
\hline 0.04 & 0.9401 & 1.4368 & 7.8226 & 1.0859 & 1.4648 & 17.0600 \\
\hline 0.06 & 0.9403 & 1.4396 & 7.8561 & 1.0865 & 1.4652 & 17.1844 \\
\hline 0.08 & 0.9409 & 1.4444 & 7.9289 & 1.0872 & 1.4668 & 17.2830 \\
\hline 0.10 & 0.9442 & 1.4468 & 8.0686 & 1.0880 & 1.4696 & 17.4708 \\
\hline \multicolumn{7}{|c|}{ ANC-6 } \\
\hline 0.01 & 0.9399 & 1.4332 & 7.6657 & 1.0847 & 1.4652 & 17.9873 \\
\hline 0.02 & 0.9423 & 1.4368 & 8.0284 & 1.0847 & 1.4656 & 18.1711 \\
\hline 0.04 & 0.9438 & 1.4420 & 8.2730 & 1.0848 & 1.4664 & 18.3289 \\
\hline 0.06 & 0.9469 & 1.4472 & 8.7974 & 1.0848 & 1.4672 & 18.4484 \\
\hline 0.08 & 0.9485 & 1.4504 & 9.2862 & 1.0860 & 1.4684 & 18.6527 \\
\hline 0.10 & 0.9514 & 1.4532 & 9.4153 & 1.0915 & 1.4696 & 18.8951 \\
\hline \multicolumn{7}{|c|}{ ANC-7 } \\
\hline 0.01 & 0.9385 & 1.4312 & 7.5152 & 1.0858 & 1.4652 & 17.8952 \\
\hline 0.02 & 0.9392 & 1.4328 & 7.6282 & 1.0867 & 1.4656 & 17.2782 \\
\hline 0.04 & 0.9405 & 1.4344 & 7.7941 & 1.0876 & 1.4676 & 18.4914 \\
\hline 0.06 & 0.9418 & 1.4380 & 7.9325 & 1.0880 & 1.4720 & 18.8069 \\
\hline 0.08 & 0.9440 & 1.4392 & 8.1349 & 1.0888 & 1.4724 & 18.9313 \\
\hline 0.10 & 0.9467 & 1.4436 & 8.4147 & 1.0895 & 1.4736 & 19.0819 \\
\hline \multicolumn{7}{|c|}{ ANC-8 } \\
\hline 0.01 & 0.9375 & 1.4324 & 7.5151 & 1.0847 & 1.4636 & 16.9630 \\
\hline 0.02 & 0.9388 & 1.4376 & 7.6369 & 1.0854 & 1.4636 & 17.0935 \\
\hline 0.04 & 0.9397 & 1.4408 & 7.6999 & 1.0859 & 1.4640 & 17.2255 \\
\hline 0.06 & 0.9410 & 1.4464 & 7.8978 & 1.0870 & 1.4644 & 17.4547 \\
\hline 0.08 & 0.9434 & 1.4484 & 8.0099 & 1.0879 & 1.4652 & 17.6120 \\
\hline 0.10 & 0.9457 & 1.4548 & 8.0975 & 1.0887 & 1.4664 & 17.8600 \\
\hline
\end{tabular}




\begin{tabular}{|l|l|l|l|l|l|l|}
\hline 0.01 & 0.9372 & 1.4316 & 7.3341 & 1.0858 & 1.4640 & 17.2055 \\
\hline 0.02 & 0.9390 & 1.4320 & 7.4198 & 1.0867 & 1.4652 & 17.8180 \\
\hline 0.04 & 0.9414 & 1.4332 & 7.4746 & 1.0871 & 1.4672 & 18.0179 \\
\hline 0.06 & 0.9448 & 1.4336 & 7.5776 & 1.0882 & 1.4676 & 18.8564 \\
\hline 0.08 & 0.9465 & 1.4368 & 7.7075 & 1.0889 & 1.4684 & 19.1683 \\
\hline 0.10 & 0.9484 & 1.4384 & 7.8073 & 1.0900 & 1.4692 & 19.4184 \\
\hline
\end{tabular}

Table 3. Some acoustical parameters of Chalocones in DMF at 308.15K.

\begin{tabular}{|c|c|c|c|c|c|}
\hline $\begin{array}{c}\text { Conc. } \\
M\end{array}$ & $\begin{array}{l}Z .10^{-5} \\
\text { g. } \mathrm{cm}^{-2}\end{array}$ & $\pi$ & $\begin{array}{c}R_{m \cdot} \cdot 10^{-3} \\
\mathrm{~cm}^{-8 / 3} \cdot \mathrm{s}^{-1 / 3}\end{array}$ & $\begin{array}{c}b \\
\mathrm{~cm}^{3} \cdot \mathrm{mol}^{-1}\end{array}$ & $r$ \\
\hline 0.00 & 1.3359 & 483.16 & 4.0943 & 78.2781 & 0.2003 \\
\hline 0.01 & 1.3417 & 481.06 & 4.1275 & 78.8983 & 0.1994 \\
\hline 0.02 & 1.3444 & 477.80 & 4.1714 & 79.7150 & 0.1981 \\
\hline 0.04 & 1.3467 & 468.20 & 4.2591 & 81.3908 & 0.1981 \\
\hline 0.06 & 1.3510 & 459.70 & 4.3528 & 83.1205 & 0.1945 \\
\hline 0.08 & 1.3539 & 451.50 & 4.4408 & 84.7848 & 0.1936 \\
\hline 0.10 & 1.3598 & 444.23 & 4.5316 & 86.4382 & 0.1891 \\
\hline \multicolumn{6}{|c|}{$A N C-2$} \\
\hline 0.01 & 1.3408 & 478.55 & 4.1326 & 78.9880 & 0.1990 \\
\hline 0.02 & 1.3444 & 473.31 & 4.1806 & 79.8543 & 0.1958 \\
\hline 0.04 & 1.3458 & 463.10 & 4.2778 & 81.6879 & 0.1945 \\
\hline 0.06 & 1.3487 & 452.15 & 4.3766 & 83.5200 & 0.1913 \\
\hline 0.08 & 1.3550 & 442.87 & 4.4737 & 85.2714 & 0.1855 \\
\hline 0.10 & 1.3626 & 434.40 & 4.5644 & 86.9027 & 0.1801 \\
\hline \multicolumn{6}{|c|}{ ANC-3 } \\
\hline 0.01 & 1.3426 & 475.98 & 4.1507 & 79.3344 & 0.1990 \\
\hline 0.02 & 1.3448 & 470.16 & 4.2160 & 80.5830 & 0.1990 \\
\hline 0.04 & 1.3477 & 453.27 & 4.3568 & 83.2434 & 0.1972 \\
\hline 0.06 & 1.3508 & 439.42 & 4.5001 & 85.9324 & 0.1945 \\
\hline 0.08 & 1.3556 & 427.49 & 4.6349 & 88.4667 & 0.1922 \\
\hline 0.10 & 1.3656 & 418.59 & 4.7420 & 90.4941 & 0.1913 \\
\hline \multicolumn{6}{|c|}{ ANC-4 } \\
\hline 0.01 & 1.3423 & 479.69 & 4.1271 & 78.8904 & 0.1994 \\
\hline 0.02 & 1.3456 & 476.38 & 4.1687 & 79.6717 & 0.1985 \\
\hline 0.04 & 1.3512 & 466.40 & 4.2562 & 81.3049 & 0.1963 \\
\hline 0.06 & 1.3583 & 458.10 & 4.3410 & 82.8722 & 0.1931 \\
\hline 0.08 & 1.3655 & 449.20 & 4.4368 & 84.5918 & 0.1868 \\
\hline 0.10 & 1.3712 & 441.04 & 4.5197 & 86.1407 & 0.1850 \\
\hline \multicolumn{6}{|c|}{ ANC-5 } \\
\hline 0.01 & 1.3427 & 490.70 & 4.1344 & 79.0157 & 0.1985 \\
\hline 0.02 & 1.3473 & 485.86 & 4.1793 & 79.8525 & 0.1972 \\
\hline 0.04 & 1.3507 & 472.60 & 4.2901 & 81.9076 & 0.1936 \\
\hline 0.06 & 1.3537 & 459.57 & 4.4009 & 83.9696 & 0.1904 \\
\hline 0.08 & 1.3590 & 448.17 & 4.5118 & 85.9897 & 0.1850 \\
\hline
\end{tabular}




\begin{tabular}{|l|l|l|l|l|l|}
\hline 0.10 & 1.3661 & 440.56 & 4.6053 & 87.7234 & 0.1823 \\
\hline \multicolumn{5}{|c|}{ ANC-6 } \\
\hline 0.01 & 1.3471 & 483.16 & 4.1281 & 78.8802 & 0.1976 \\
\hline 0.02 & 1.3539 & 489.51 & 4.1791 & 79.7892 & 0.1936 \\
\hline 0.04 & 1.3610 & 493.06 & 4.2937 & 81.8774 & 0.1877 \\
\hline 0.06 & 1.3704 & 484.39 & 4.3997 & 83.7989 & 0.1819 \\
\hline 0.08 & 1.3757 & 484.50 & 4.5103 & 85.8429 & 0.1783 \\
\hline 0.10 & 1.3826 & 472.97 & 4.6129 & 87.7378 & 0.1751 \\
\hline \multicolumn{7}{|c|}{ ANC-7 } \\
\hline 0.01 & 1.3432 & 486.00 & 4.1217 & 78.7942 & 0.1999 \\
\hline 0.02 & 1.3457 & 483.06 & 4.1680 & 79.6499 & 0.1981 \\
\hline 0.04 & 1.3491 & 475.72 & 4.2592 & 81.3624 & 0.1963 \\
\hline 0.06 & 1.3543 & 467.56 & 4.3519 & 83.0651 & 0.1922 \\
\hline 0.08 & 1.3586 & 462.30 & 4.4373 & 84.6705 & 0.1909 \\
\hline 0.10 & 1.3667 & 459.04 & 4.5225 & 86.2097 & 0.1859 \\
\hline \multicolumn{7}{|c|}{ ANC-8 } \\
\hline 0.01 & 1.3429 & 484.72 & 4.1325 & 78.9796 & 0.1985 \\
\hline 0.02 & 1.3496 & 480.98 & 4.1849 & 79.8847 & 0.1927 \\
\hline 0.04 & 1.3539 & 468.81 & 4.2902 & 81.8342 & 0.1891 \\
\hline 0.06 & 1.3611 & 461.02 & 4.3957 & 83.7380 & 0.1828 \\
\hline 0.08 & 1.3664 & 452.11 & 4.4914 & 85.5216 & 0.1805 \\
\hline 0.10 & 1.3758 & 442.29 & 4.5914 & 87.2964 & 0.1733 \\
\hline \multicolumn{7}{|c|}{ ANC-9 } \\
\hline 0.01 & 1.3417 & 476.92 & 4.1476 & 79.2824 & 0.1994 \\
\hline 0.02 & 1.3446 & 471.28 & 4.2075 & 80.4197 & 0.1990 \\
\hline 0.04 & 1.3492 & 456.56 & 4.3321 & 82.7784 & 0.1976 \\
\hline 0.06 & 1.3545 & 444.76 & 4.4495 & 85.0138 & 0.1972 \\
\hline 0.08 & 1.3599 & 433.48 & 4.5772 & 87.3901 & 0.1936 \\
\hline 0.10 & 1.3642 & 422.39 & 4.7014 & 89.7271 & 0.1918 \\
\hline
\end{tabular}

Table 4. Some acoustical parameters of Chalcones in DMSO at $303.15 \mathrm{~K}$.

\begin{tabular}{|c|c|c|c|c|c|}
\hline $\begin{array}{c}\text { Conc. } \\
M\end{array}$ & $\begin{array}{l}Z .10^{-5} \\
\text { g. } \mathrm{cm}^{-2}\end{array}$ & $\pi$ & $\begin{array}{c}R_{m} \cdot 10^{-3} \\
\mathrm{~cm}^{-8 / 3} \cdot \mathrm{s}^{-1 / 3}\end{array}$ & $\begin{array}{c}b \\
\mathrm{~cm}^{3} \cdot \mathrm{mol}^{-1}\end{array}$ & $r$ \\
\hline \multicolumn{6}{|c|}{$A N C-1$} \\
\hline 0.00 & 1.5839 & 737.58 & 3.7935 & 72.0341 & 0.1669 \\
\hline 0.01 & 1.5845 & 738.52 & 3.8286 & 72.6949 & 0.1664 \\
\hline 0.02 & 1.5851 & 732.84 & 3.8638 & 73.3554 & 0.1660 \\
\hline 0.04 & 1.5871 & 721.00 & 3.9348 & 74.6757 & 0.1641 \\
\hline 0.06 & 1.5877 & 707.62 & 4.0035 & 75.9803 & 0.1641 \\
\hline 0.08 & 1.5887 & 694.55 & 4.0725 & 77.2830 & 0.1637 \\
\hline 0.10 & 1.5937 & 683.95 & 4.1416 & 78.5366 & 0.1600 \\
\hline \multicolumn{6}{|c|}{$A N C-2$} \\
\hline 0.01 & 1.5925 & 754.60 & 3.8351 & 72.6978 & 0.1582 \\
\hline 0.02 & 1.5935 & 749.73 & 3.8711 & 73.3679 & 0.1573 \\
\hline 0.04 & 1.5993 & 737.40 & 3.9438 & 74.6715 & 0.1522 \\
\hline
\end{tabular}




\begin{tabular}{|c|c|c|c|c|c|}
\hline 0.06 & 1.6024 & 724.48 & 4.0132 & 75.9571 & 0.1504 \\
\hline 0.08 & 1.6043 & 712.07 & 4.0808 & 77.2307 & 0.1499 \\
\hline 0.10 & 1.6078 & 704.37 & 4.1486 & 78.4838 & 0.1481 \\
\hline \multicolumn{6}{|c|}{$A N C-3$} \\
\hline 0.01 & 1.5922 & 752.25 & 3.8522 & 73.0298 & 0.1587 \\
\hline 0.02 & 1.5976 & 745.41 & 3.9082 & 74.0173 & 0.1536 \\
\hline 0.04 & 1.6034 & 727.26 & 4.0136 & 75.9511 & 0.1495 \\
\hline 0.06 & 1.6134 & 715.86 & 4.1211 & 77.8600 & 0.1411 \\
\hline 0.08 & 1.6265 & 710.01 & 4.2166 & 79.5491 & 0.1337 \\
\hline 0.10 & 1.6358 & 695.34 & 4.3138 & 81.3024 & 0.1286 \\
\hline \multicolumn{6}{|c|}{$A N C-4$} \\
\hline 0.01 & 1.5892 & 739.33 & 3.8324 & 72.7008 & 0.1619 \\
\hline 0.02 & 1.5925 & 736.12 & 3.8646 & 73.2909 & 0.1605 \\
\hline 0.04 & 1.5949 & 722.68 & 3.9323 & 74.5679 & 0.1600 \\
\hline 0.06 & 1.6001 & 712.03 & 4.0026 & 75.8460 & 0.1564 \\
\hline 0.08 & 1.6032 & 709.73 & 4.0677 & 77.0728 & 0.1559 \\
\hline 0.10 & 1.6054 & 701.03 & 4.1332 & 78.3145 & 0.1559 \\
\hline \multicolumn{6}{|c|}{$A N C-5$} \\
\hline 0.01 & 1.5876 & 733.33 & 3.8363 & 72.7945 & 0.1632 \\
\hline 0.02 & 1.5881 & 727.86 & 3.8767 & 73.5546 & 0.1628 \\
\hline 0.04 & 1.5906 & 712.66 & 3.9541 & 75.0091 & 0.1619 \\
\hline 0.06 & 1.5919 & 698.79 & 4.0328 & 76.4949 & 0.1614 \\
\hline 0.08 & 1.5947 & 684.76 & 4.1121 & 77.9696 & 0.1596 \\
\hline 0.10 & 1.5989 & 672.81 & 4.1919 & 79.4325 & 0.1564 \\
\hline \multicolumn{6}{|c|}{$A N C-6$} \\
\hline 0.01 & 1.5893 & 757.43 & 3.8408 & 72.8522 & 0.1614 \\
\hline 0.02 & 1.5897 & 751.26 & 3.8846 & 73.6768 & 0.1609 \\
\hline 0.04 & 1.5908 & 735.12 & 4.9719 & 75.3189 & 0.1600 \\
\hline 0.06 & 1.5916 & 718.91 & 4.0596 & 76.9680 & 0.1591 \\
\hline 0.08 & 1.5947 & 705.54 & 4.1427 & 78.5228 & 0.1577 \\
\hline 0.10 & 1.6041 & 695.60 & 4.2072 & 79.7230 & 0.1564 \\
\hline \multicolumn{6}{|c|}{$A N C-7$} \\
\hline 0.01 & 1.5909 & 757.85 & 3.8288 & 72.6254 & 0.1614 \\
\hline 0.02 & 1.5927 & 758.08 & 3.8613 & 73.2344 & 0.1609 \\
\hline 0.04 & 1.5962 & 746.46 & 3.9303 & 74.5100 & 0.1587 \\
\hline 0.06 & 1.6015 & 736.44 & 4.0033 & 75.8180 & 0.1536 \\
\hline 0.08 & 1.6031 & 724.26 & 4.0710 & 77.0937 & 0.1531 \\
\hline 0.10 & 1.6055 & 712.78 & 4.1397 & 78.3733 & 0.1518 \\
\hline \multicolumn{6}{|c|}{$A N C-8$} \\
\hline 0.01 & 1.5876 & 736.87 & 3.8353 & 72.7740 & 0.1632 \\
\hline 0.02 & 1.5886 & 731.27 & 3.8720 & 73.4722 & 0.1632 \\
\hline 0.04 & 1.5898 & 717.22 & 3.9491 & 74.9274 & 0.1628 \\
\hline 0.06 & 1.5918 & 706.01 & 4.0237 & 76.3354 & 0.1623 \\
\hline 0.08 & 1.5940 & 693.65 & 4.0991 & 77.7528 & 0.1614 \\
\hline 0.10 & 1.5965 & 683.38 & 4.1752 & 79.1734 & 0.1600 \\
\hline \multicolumn{6}{|c|}{$A N C-9$} \\
\hline 0.01 & 1.5896 & 740.09 & 3.8425 & 72.9045 & 0.1628 \\
\hline
\end{tabular}




\begin{tabular}{|l|l|l|l|l|l|}
\hline 0.02 & 1.5922 & 741.99 & 3.8903 & 73.7917 & 0.1614 \\
\hline 0.04 & 1.5950 & 724.07 & 3.9906 & 75.6593 & 0.1591 \\
\hline 0.06 & 1.5970 & 720.10 & 4.0865 & 77.4706 & 0.1587 \\
\hline 0.08 & 1.5989 & 706.04 & 4.1841 & 79.3062 & 0.1577 \\
\hline 0.10 & 1.6014 & 691.75 & 4.2797 & 81.1039 & 0.1568 \\
\hline
\end{tabular}

Table 5. The Bachem's constants $A$ and $B, \phi_{k}^{o}$ and $S_{k}, \Phi_{V}^{O}$ and $S_{v}$ of Chalcones in DMF and DMSO at $303.15 \mathrm{~K}$.

\begin{tabular}{|c|c|c|c|c|c|c|}
\hline Comp. & $\begin{array}{c}A X 10^{11} \\
d y n^{-1} \cdot \mathrm{cm}^{3} \\
\mathrm{~mol}^{-1}\end{array}$ & $\begin{array}{c}B X 10^{I I} \\
d y n^{-1} \cdot \mathrm{cm}^{-1 / 2} \\
\mathrm{~mol}^{-3 / 2}\end{array}$ & $\begin{array}{c}\phi_{k}^{o} X 10^{8} \\
d y n^{-1} \cdot m^{-1}\end{array}$ & $\begin{array}{c}S_{k} \times 10^{8} \\
d y n^{-1} \mathrm{~cm}^{-3 / 2} \\
\mathrm{~mol}^{-3 / 2}\end{array}$ & $\begin{array}{c}\Phi_{V}^{O} \\
\mathrm{~cm}^{2} . \mathrm{mol}^{-1}\end{array}$ & $\begin{array}{c}S_{v} \\
\mathrm{~cm}^{2} \cdot \mathrm{dm}^{1 / 2} \\
. \mathrm{mol}^{-3 / 2}\end{array}$ \\
\hline \multicolumn{7}{|c|}{$D M F$} \\
\hline$A N C-1$ & -1.70 & 2.04 & -0.80 & 2.70 & -124.0 & 1040.00 \\
\hline$A N C-2$ & -2.56 & 3.53 & -3.00 & 10.60 & -44.0 & 1777.78 \\
\hline$A N C-3$ & -2.40 & 4.60 & -1.30 & 5.93 & -290.0 & 4206.89 \\
\hline$A N C-4$ & -2.40 & 3.24 & -1.75 & 3.00 & -176.0 & 975.00 \\
\hline$A N C-5$ & -4.25 & 10.71 & -5.00 & 1.65 & -386.0 & 7142.85 \\
\hline$A N C-6$ & -4.60 & 6.33 & -5.40 & 12.50 & -262.0 & 1900.00 \\
\hline$A N C-7$ & -3.60 & 9.09 & -1.74 & 4.89 & -74.0 & 225.35 \\
\hline$A N C-8$ & -3.56 & 4.76 & -2.32 & 1.56 & -57.0 & 178.08 \\
\hline$A N C-9$ & -2.20 & 3.44 & -1.20 & 3.47 & -164.0 & 1027.00 \\
\hline \multicolumn{7}{|c|}{ DMSO } \\
\hline$A N C-1$ & -0.26 & 0.10 & -0.35 & 10.36 & 71.00 & 127.27 \\
\hline$A N C-2$ & -3.65 & 8.75 & -2.35 & 7.82 & 53.00 & 150.00 \\
\hline$A N C-3$ & -4.60 & 7.45 & -2.85 & 11.40 & 55.00 & 716.66 \\
\hline$A N C-4$ & -2.88 & 6.53 & -1.26 & 5.25 & -13.00 & 410.71 \\
\hline$A N C-5$ & -1.60 & 3.73 & -0.03 & 0.38 & 43.00 & 61.53 \\
\hline$A N C-6$ & -2.75 & 8.64 & -1.14 & 7.68 & 65.50 & 266.66 \\
\hline$A N C-7$ & -2.90 & 7.29 & -1.22 & 4.10 & -46.60 & 928.57 \\
\hline$A N C-8$ & -1.00 & 1.76 & -0.34 & 1.18 & 35.00 & 425.00 \\
\hline$A N C-9$ & -2.06 & 4.32 & -1.08 & 2.62 & 9.00 & 220.93 \\
\hline
\end{tabular}




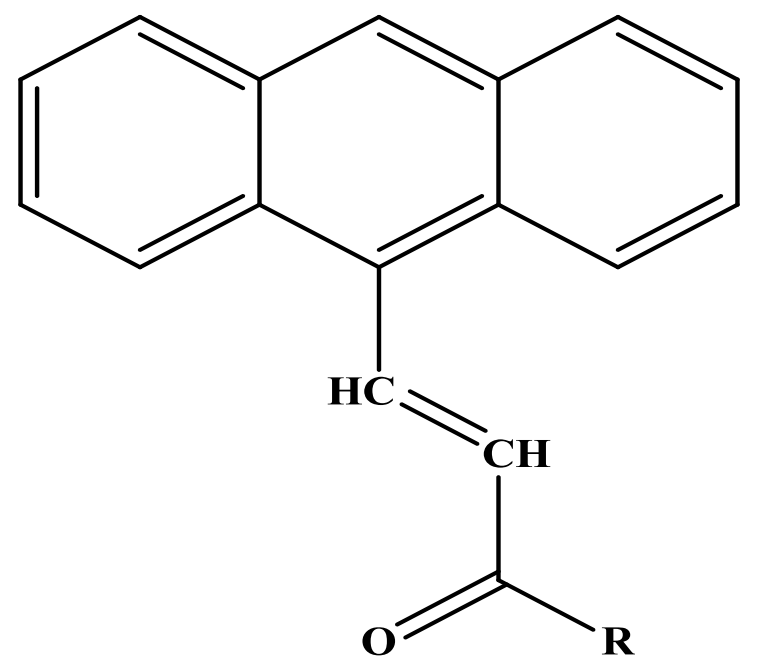

$$
\mathbf{R}=\text { Aryl }
$$

Fig. 1. General structure of Chalcone.
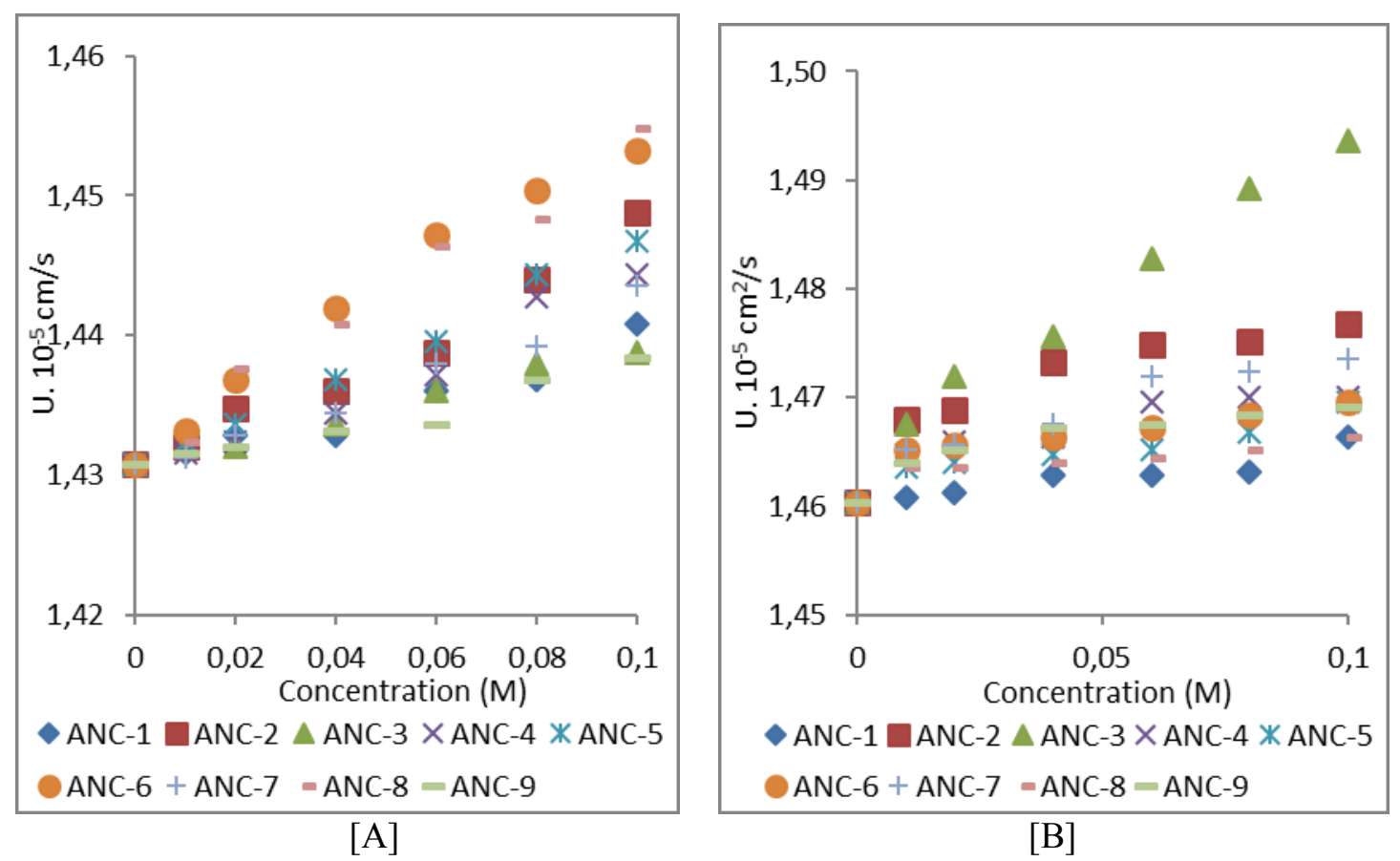

Fig. 2. Variation of ultrasonic velocity with concentration of Chalcones in [A] DMF and [B] DMSO at $303.15 \mathrm{~K}$. 


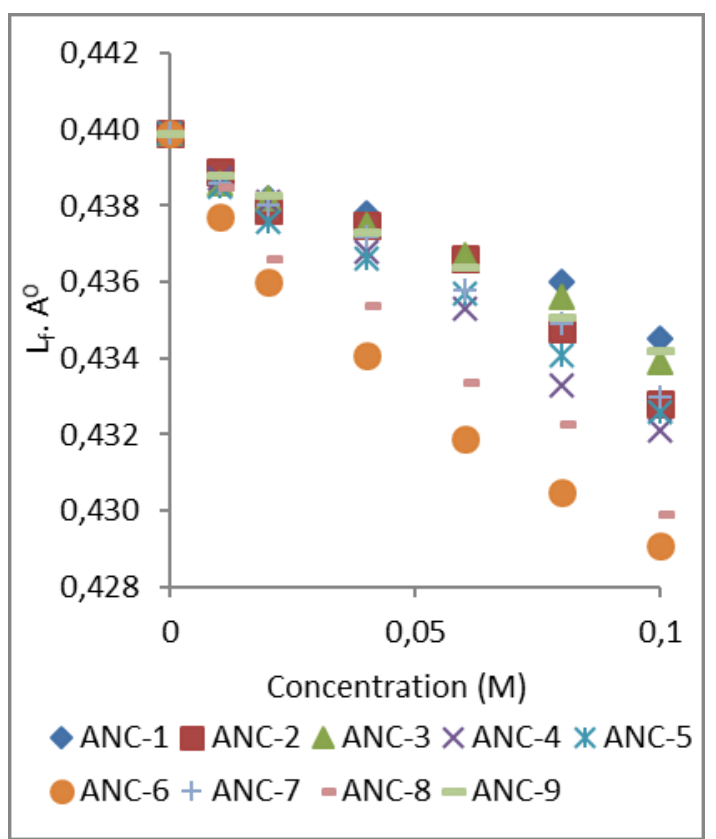

[A]

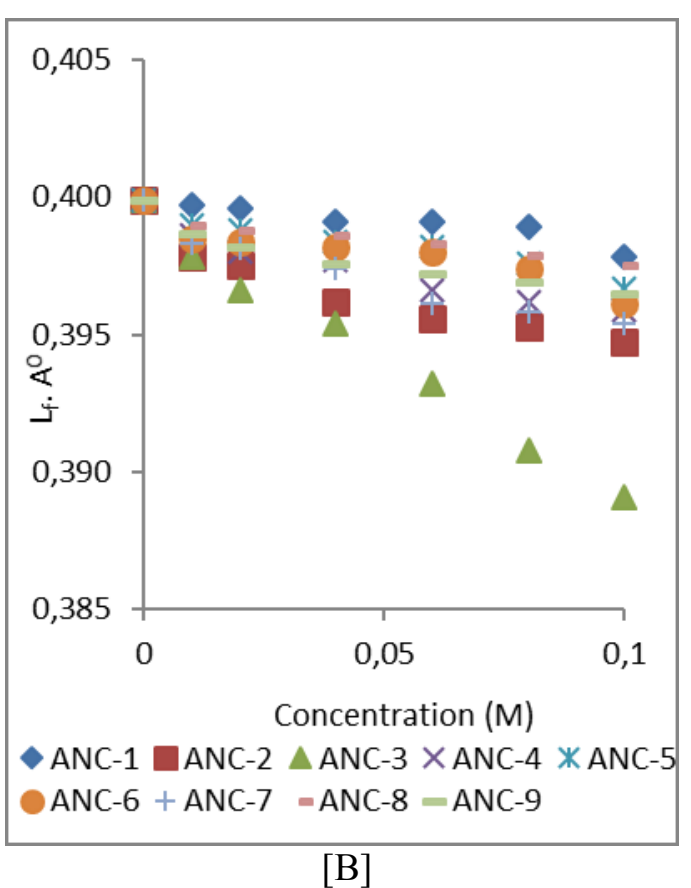

Fig. 3. Variation of intermolecular free path length $\left(\mathrm{L}_{\mathrm{f}}\right)$ with concentration of Chalcones in $[A]$ DMF and [B] DMSO at $303.15 \mathrm{~K}$.

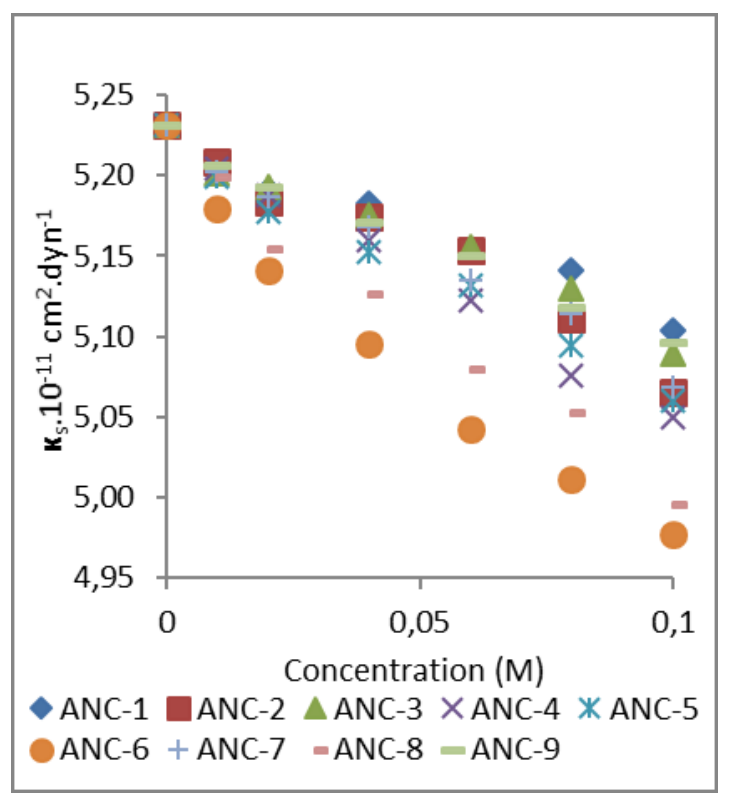

$[\mathrm{A}]$

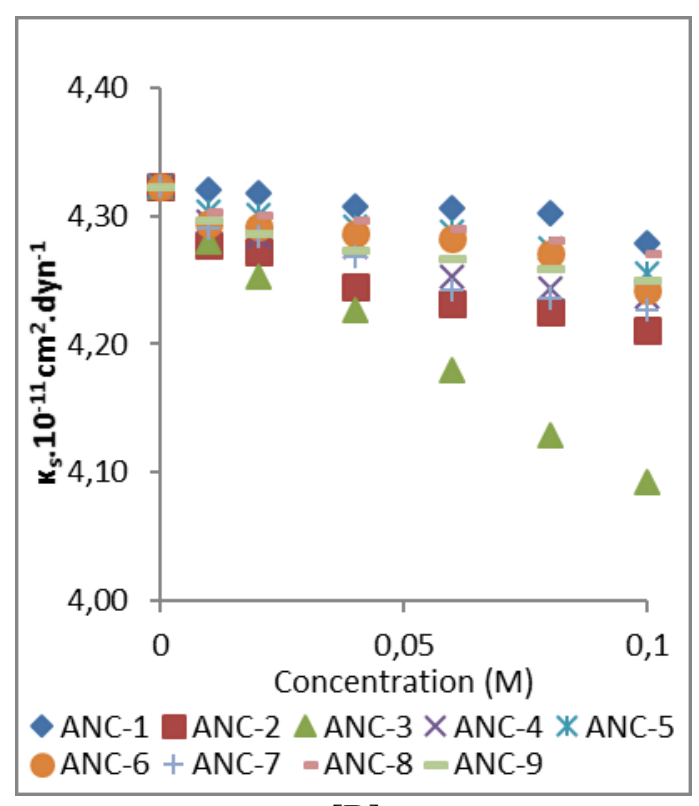

[B]

Fig. 4. Variation of adiabatic compressibility $\left(\kappa_{\mathrm{s}}\right)$ with concentration of of Chalcones in $[\mathrm{A}] \mathrm{DMF}$ and [B] DMSO at $303.15 \mathrm{~K}$. 


\section{References}

[1] S. J. McNeil, R. A. McCall, Ultrasonic Sonochemistry 18 (2011) 401-406.

[2] M. Foguel, C. Uliana, U. Tomaz, O. Marques, H. Yamanaka, A. Ferreira, Ecletica Quimica. 34(2009) 59-66.

[3] S. Nanda, R. Rao, S. Anand, Indian Pharma 9(2010) 49-54.

[4] G. Hong-xia, G. Xiu-qiong, B. Hong-ru, K. Wang, Yinran Zhuji 27(2010) 34-35.

[5] N. Buyukkamaci, A. Filibeli, G. Erden, Desalination. 259(2010) 223-227.

[6] R. Katoh, H. Yokoi, S. Usuba, Y. Kakudate, S. Fujiwara, Ultrasonic Sonochemistry 5(1998) 69-72.

[7] M. Mexiarova, T. Kiripolsky, Ultrasonic Sonochemistry 12(2005) 401-403.

[8] M. Patil, V. Onuora V., Injury 25(1994) 177-178.

[9] I. Lovett, B. Doust, N. Orr, Australian Radiology. 32(1988) 104-106.

[10] A. Boucaud, International Journal of Pharmaceutics 228(2001) 69-77.

[11] N. Inoue, T. Hasegawa, K. Matsuzawa, K. Acustica 74(1991) 128-133.

[12] T. Takagi, Review of Physical Chemistry 48(1978) 10-16.

[13] J. Nath, A. Dixit, Journal of Chemical Engineering Data 29(1984) 320-321.

[14] S. Raman, C. Tipnis, Indian Journal of Pure and Applied Physics 20(1982) 79-80.

[15] M. H. Lim, S. H. Kim, Y. U.Kim, J. Khim, Ultrasonic Sonochemistry 14(2007) 93-98.

[16] D. Laux, G. Leveque, C. Cereser, C., Ultrasonics 49(2009) 159-161.

[17] S. Hassun, A. Shihab, F. Jassim, Chinese Journal of Polymer Science 7(1989) 270279.

[18] S. Srivastava, S. Laxmi, Zeitschrift für Physikalische Chemie 70(1970) 219-223.

[19] P. Sharma, S. Chauhan, M. Chauhan, V. Syal, Indian Journal of Pure and Applied Physics 46(2008)839-843.

[20] S. Baluja Chinese Journal of Chemistry 24(2006) 1327-1331.

[21] S. Baluja, A. Solanki, N. Kachhadia, Physics and Chemistry of Liquids 45(2007) 561569.

[22] R. Gajera, R. Bhalodia, S. Baluja, International Journal of Applied Chemistry 5(2009) 47-55.

[23] J. Riddick, W. Bunger, T. Sakano,"Organic solvents-Physical properties and Methods for purification, 4th Edition, Techniques of Chemistry, Vol.II, Wiely, New York 1986.

[24] C. H. Bachem, Physikalische Zeitschrift 101(1936) 541-577.

[25] F. T. Gucker, Chemical Review 13(1933) 111-130. 